\title{
9-Fold Fresnel Köhler Concentrator For Increased Uniform Irradiance On High Concentrations
}

\author{
João Mendes-Lopes , Pablo Benítez , Pablo Zamora and Juan Carlos Miñano
}

\begin{abstract}
Non-uniform irradiance patterns created by Concentrated Photovoltaics (CPV) concentrators over Multi-Junction Cells (MJC) can originate significant power losses, especially when there are different spectral irradiance distributions over the different MJC junctions. This fact has an increased importance considering the recent advances in 4 and 5 junction cells. The spectral irradiance distributions are especially affected with thermal effects on Silicone-on-Glass (SoG) CPV systems. This work presents a new CPV optical design, the 9-fold Fresnel Köhler concentrator, prepared to overcome these effects at high concentrations while maintaining a large acceptance angle, paving the way for a future generation of high efficiency CPV systems of 4 and 5 junction cells.
\end{abstract}

Keywords: Nonimaging, concentrator, photovoltaic, solar energy, Köhler integration, SoG, irradiance distribution

\section{INTRODUCTION}

Concentrator Photovoltaic (CPV) systems are presented as a solution to achieve high efficiency on the conversion of Sun light into electric power, by concentrating light into a small Multi-Junction cell (MJ) using low cost optical materials. The optics systems have to ensure a good optical efficiency, large acceptance angle, and good irradiance distribution on the cell, while maintaining a high concentration $\mathrm{C}_{\mathrm{g}}$. The acceptance angle $(\alpha)$ provides tolerance against tracking misalignments and manufacturing errors, and has a tradeoff with the concentration. This tradeoff is described through the concentration-acceptance angle product CAP, defined by $C_{g}{ }^{1 / 2} \sin \alpha$, being an appropriate merit function for a concentrator ${ }^{1}$.

The irradiance distribution over the PV cell is also fundamental for high efficiency, due to the fact that non-uniform patterns can cause an increase in the effective series resistance of the cell, creating a drop in efficiency. In particular, the typical non-uniform irradiance patterns created by conventional CPV concentrators over MJ cells can originate significant power losses. Furthermore, when chromatic aberrations between irradiance distributions over the different junctions are significant, the effect on power losses increases considerably ${ }^{2-4}$.

The advances in 4 and 5 junction solar cells suggests that the spectrum balance and irradiance uniformity between different junctions will be increasingly important for future CPV systems. ${ }^{5}$. These cells are likely to be more expensive, therefore requiring higher concentration to be cost effective.

The most recent optical designs for CPV systems, namely Fresnel-Köhler (for this work, presented as FK4) technology, have proven that spectral balanced high irradiance uniformity is achievable, while maintaining a high acceptance angle, for concentrations of 850-1000x $x^{6}$. However, when aiming for larger concentrations, irradiance uniformity decreases for a given architecture, as demonstrated in ${ }^{4}$ (for SILO system, which has almost perfect uniformity at $300 \mathrm{x}^{7}$ ).

Interest on Silicone-on-Glass (SoG) lens has been growing due to combining mass production simplicity and high resistance to external factors. SoG lens are affected by thermal changes, with a refractive index variation of $-0.00036 /{ }^{\circ} \mathrm{C}$, according to ${ }^{8}$. This variation has a considerable effect on spectral balance of irradiance distributions.

This work presents a new CPV optical design, 9-fold Fresnel-Köhler (FK9) concentrator, prepared to overcome these effects while maintaining a large acceptance angle, paving the way for 4 and 5 junction based CPV systems at higher concentrations. 

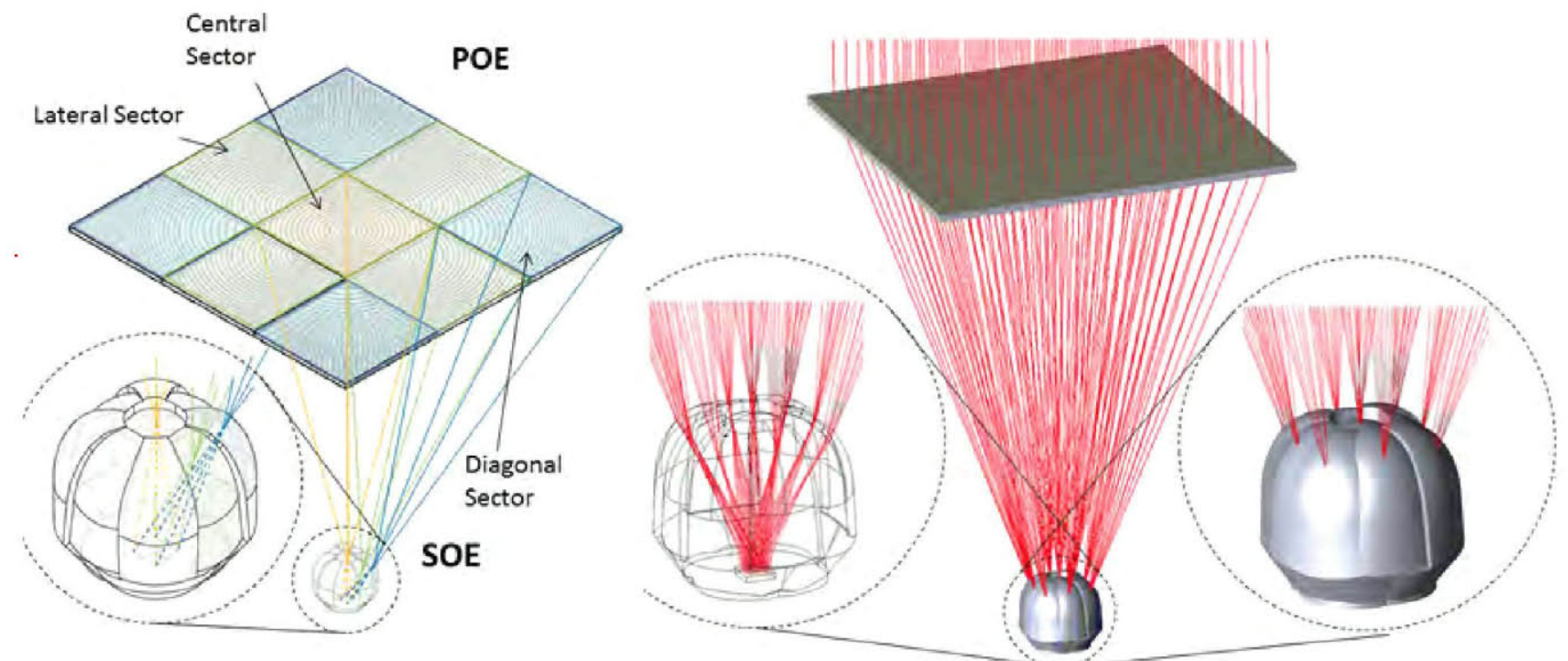

Figure 1 on the left - Schematic view of FK9 concentrator. Three different fold types, central, lateral and diagonal (in the picture, orange, green and blue, respectively) form a 9 fold system. Each sector of the SOE images the corresponding sector of the POE in the cell. on the right - a FK9 ray tracing simulation, where the 9 focus on SOE sectors are visible, as well as the image on the cell

\section{TECHNICAL DESCRIPTION OF FK9}

FK9 consists on a Primary Optical Element (POE), a Fresnel lens divided in 9 sectors, and a Secondary Optical Element (SOE) also divided in 9 sectors, all of them free form, and coupled with the respective sector of the POE, as seen in Figure 1. The POE consists in 1 symmetric central sub Fresnel lens, four lateral and for diagonal sub Fresnel lenses, each of them symmetric with each other relative to the Fresnel lens center. The four lateral and four diagonal sub-lenses may be seen as off-center square pieces of a symmetric Fresnel lens.

The SOE has the same structure: 1 central sub-lens, four lateral and four diagonal sub-lenses, all symmetric with each other relative to the center axis, all of them free form. Each sub-lens is coupled with a sector of the POE, imaging each square sector of the Fresnel lens into the cell, thus performing Köhler integration. The integration allows an excellent spectrum and spatial irradiance on the cell, even at very high concentrations and extreme thermic conditions, as presented below. The fact that the incoming bundle of Sun rays is divided into nine channels enables a very high acceptance for rays coming from any direction, thus allowing a high CAP.

Table 1. 9 Fold Fresnel Köhler concentrator simulation results for $\mathrm{C}_{\mathrm{g}}=1000 \mathrm{x}$

\begin{tabular}{cc}
\hline $\mathbf{C}_{\mathrm{g}}=\mathbf{1 0 0 0 x}$ & Simulation results \\
\hline Optical Efficiency & $83.9 \%$ \\
Acceptance angle & $\pm 1.17^{\circ}$ \\
CAP & 0.65 \\
f-number & 1 \\
\hline
\end{tabular}

\section{SIMULATION RESULTS FOR $\mathrm{C}_{\mathrm{G}}=1000 \mathrm{X}$}

Simulation results are presented for a FK9 designed for a geometrical concentration of $\mathbf{C}_{\mathrm{g}}=\mathbf{1 0 0 0 X}$ (over cell illuminated area) and an F-number of $\mathbf{1}$. All the following simulation results correspond to raytracing simulations carried out under the same realistic conditions: AM1.5D sunlight spectrum, finite sun $\left( \pm 0.265^{\circ}\right)$ and Fresnel and absorption losses. POE is made on PMMA, and SOE is made on Schott B270 glass. Moreover, no AR coating has been applied on SOE. POE teeth rounded edges were not considered.

Table 1 summarizes the main results of FK9 optical performance, and Figure 2 presents its acceptance curve. 1000x FK9 has an acceptance angle of $\pm 1.17^{\circ}$. Also, the curve almost maintains its maximum efficiency until it reaches $1^{\circ}$ of 
deviation, ensuring a very high efficiency within the acceptance angle. The concentration level of $\mathrm{C}_{\mathrm{g}}=1000 \mathrm{x}$ and an acceptance angle of $\pm 1.17^{\circ}$ represents a CAP of 0.65 .

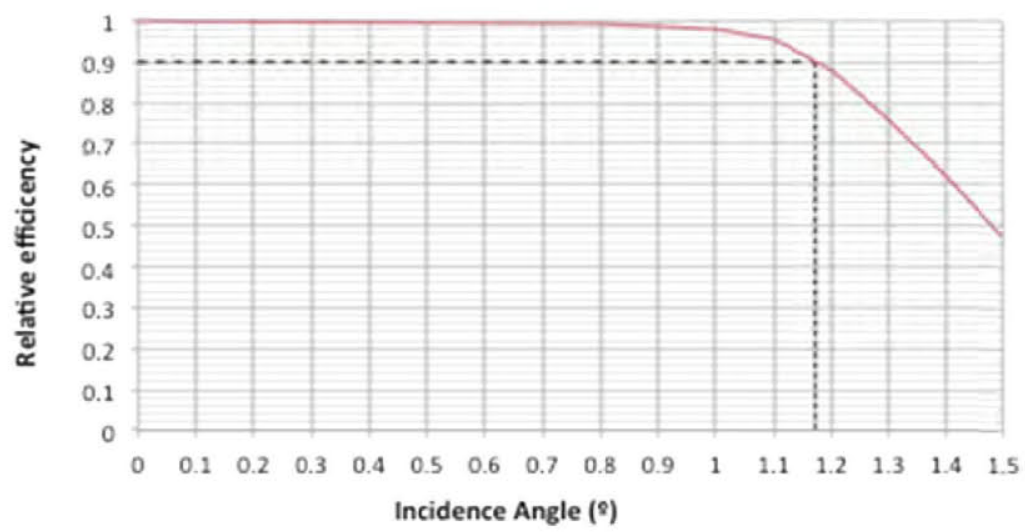

Figure 2. FK 9 acceptance curve for $\mathrm{C}_{\mathrm{g}}=1000 \mathrm{x}$. The acceptance angle is $\pm 1.17^{\circ}$

Figure 3 represents the spectral irradiance distributions on each junction of a triple MJ cell that FK9 produces at normal incidence. The spatial irradiance is almost perfectly uniform for all junctions, and this uniform spatial irradiance is kept for the full Sun light spectrum. This is especially important to avoid current mismatches between junctions and high series resistant losses, and even more if one considers cells with 4 and 5 junctions, as they are more likely to be sensitive to spectrum variations, thus it is more difficult to achieve current mismatch.

Figure 4 presents a CAP comparison between different systems, in which all of them were designed for achieving the most uniform spatial and spectral irradiance possible. While FK4 and FK9 are the systems with highest performance in this field, FK9 still outperforms the FK4 concentrator, presenting a higher CAP of 0.65 and higher acceptance angle, therefore reaching a higher tolerance value.

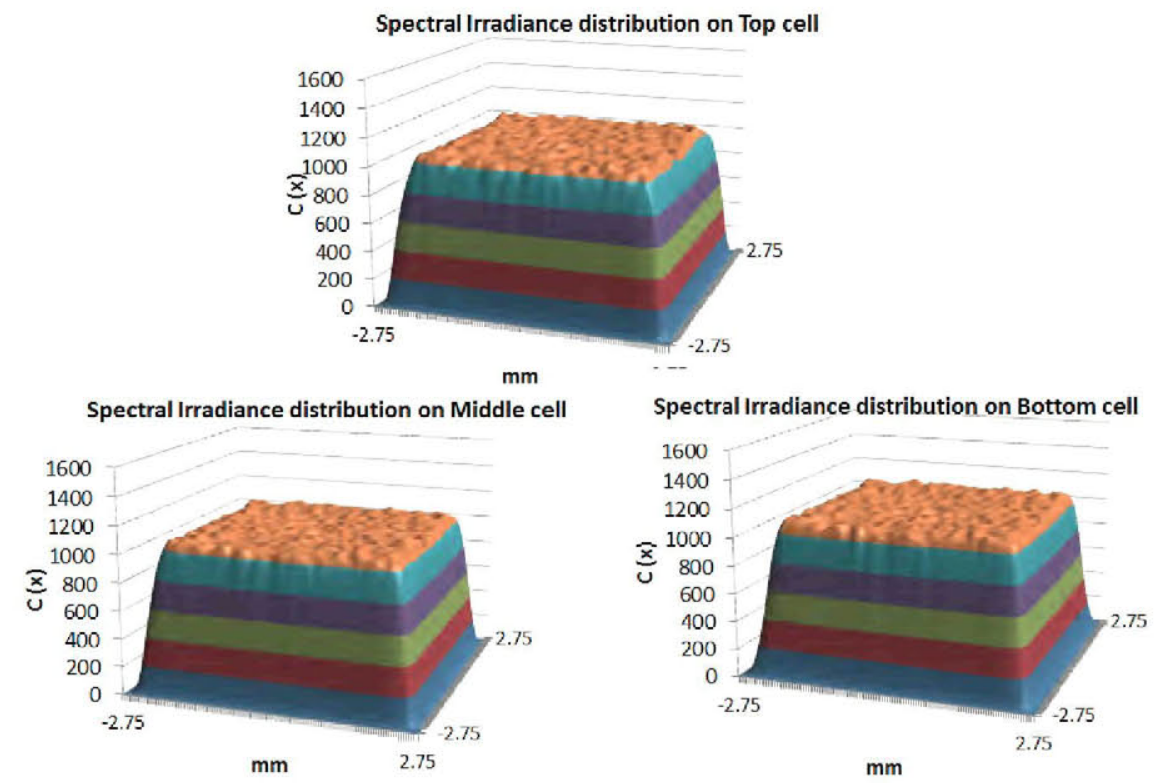

Figure 3. 1000x FK9 spectral irradiance distributions for Top, Middle, and Bottom junction of a Triple MJ cell. The irradiance distribution throughout the full Sun light spectrum 


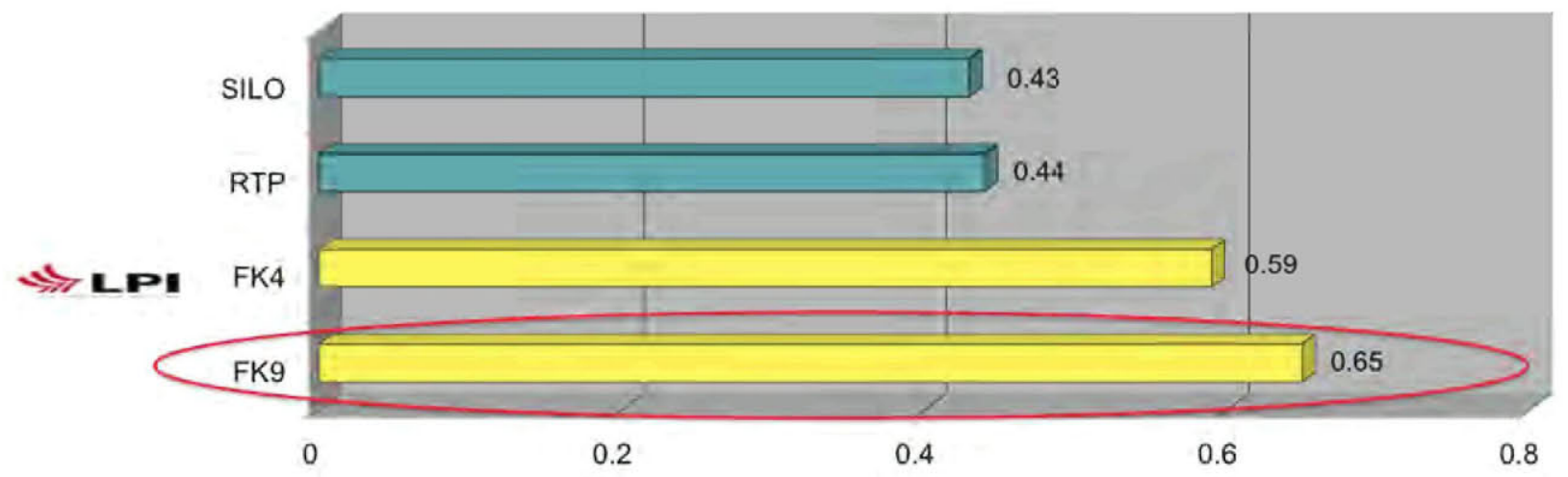

Figure 4. Comparison of CAP between different systems with rather good to excellent irradiance uniformity

\subsection{Spectral irradiance distributions at high concentrations and high temperatures}

To analyze the effects of high concentrations and thermal variations in SoG lens based CPV systems on the spectrum irradiance distributions, a comparative study between three different SoG lens-based systems was made to evaluate their irradiance and spectral maps when designed for $1000 \mathrm{x}$ and $2000 \mathrm{x}$ concentration ratio, at two different $\mathrm{POE}, 20^{\circ} \mathrm{C}$ and $45^{\circ} \mathrm{C}$. The analyzed systems were the Silo, FK4 and FK9 (FIGURE 5). These systems were chosen for their capability of creating uniform spectral irradiance distributions at a specific concentration level. All three systems were designed with a SoG lens as POE, and an SOE made of b270 glass. The systems were designed for a POE temperature of $20^{\circ} \mathrm{C}$.

The temperature effects on SoG lens were defined according to [8]. No thermal expansion was taken into account. For a difference of $25^{\circ} \mathrm{C}$, a decrease of -0.009 is considered for SoG lens refractive index. On Bottom and Top cell irradiance distributions are presented to represent the most extreme cases.

All results are presented in Figure 5. It can be seen, that for $1000 \mathrm{x}$ at $\mathrm{T}_{\mathrm{POE}}=20^{\circ} \mathrm{C}$, the SILO already presents a nonuniform irradiance, especially at top cell. For this same concentration, when $\mathrm{T}_{\mathrm{POE}}$ increases to $45^{\circ} \mathrm{C}$, the refractive index variation causes a considerable change in bottom cell irradiance distribution. Considering $\mathrm{C}_{\mathrm{g}}=2000 \mathrm{X}$, this effect is even clearer: for $\mathrm{T}_{\mathrm{POE}}=20^{\circ} \mathrm{C}$, the non-uniformity has increased considerably in both junctions, only due to the concentration increase. If we analyze an even more extreme case of $\mathrm{C}_{\mathrm{g}}=2000 \mathrm{x}$ and $\mathrm{T}_{\mathrm{POE}}=45^{\circ} \mathrm{C}$, the non uniformity in bottom cell irradiance has a dramatic change.

FK4 and FK9, as expected and presented previously, have an excellent spectral irradiance distribution for both cells, at $1000 \mathrm{x}$ and $\mathrm{T}_{\mathrm{POE}}=$ of $20^{\circ} \mathrm{C}$. When temperature increases to $45^{\circ} \mathrm{C}$, FK4 bottom cell irradiance uniformity suffers a slight decrease, while the FK9 maintains the irradiance uniformity in both junctions.

When $\mathrm{C}_{\mathrm{g}}=2000 \mathrm{x}$ is considered, while both FK4 and FK9 still maintain an almost excellent uniformity for both junction at $\mathrm{T}_{\mathrm{POE}}=20^{\circ} \mathrm{C}$, for $\mathrm{T}_{\mathrm{POE}}=45^{\circ} \mathrm{C}$, FK4 suffers a significant non uniform variation for the Bottom cell irradiance, while FK9 uniformity variation in both junctions is almost negligible, maintaining an excellent uniformity. 


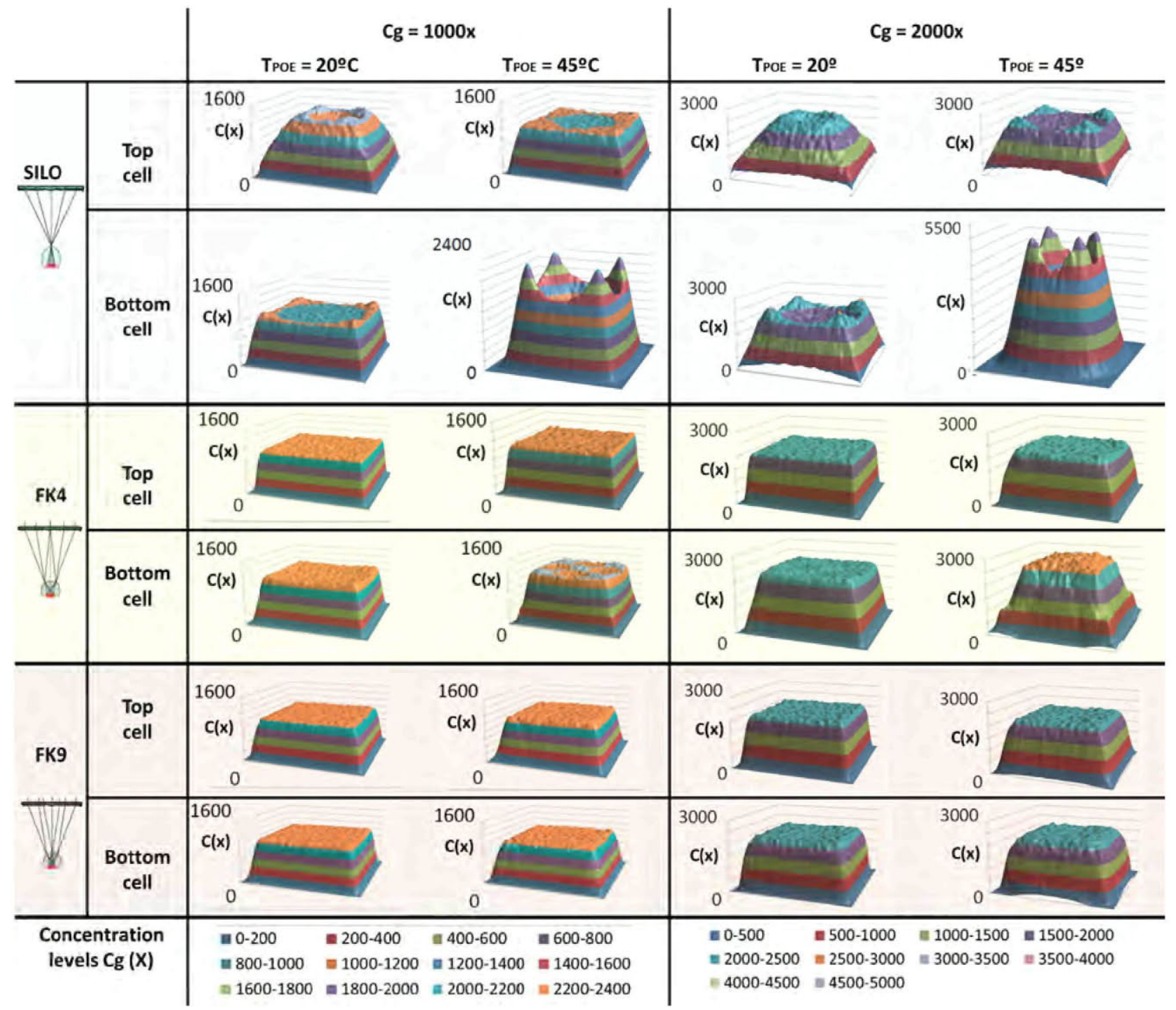

Figure 5. Spectral Irradiance distributions on Top and Bottom cell for three different CPV systems well known for their excellent irradiance uniformity at specific concentrations

\section{CONCLUSIONS}

A new CPV system, based on a 9-fold Fresnel-Köhler concentrator (FK9), was presented, with a CAP of 0.65, with $\pm 1.17^{\circ}$ for $\mathrm{C}_{\mathrm{g}}=1000 \mathrm{x}$. The system presents an excellent spatial and spectrum irradiance distribution for each junction of a Triple MJ cell.

A comparative study showed not only that high concentration levels and high temperatures (on SoG lens based systems) affect significantly the spectral irradiance distributions, but also that the design of FK9 is prepared to overcome these aberration effects, maintaining an excellent uniformity for all junctions even in extreme conditions.

This capability is decisive for 4 and 5 junction cells, due to the fact that these cells are likely to require high spectral irradiance uniformity and high concentration for an efficient and cost effective system.

FK9 paves the way for the future generation of high efficiency CPV systems of 4 and 5 junction cells. 


\section{ACKNOWLEDGMENTS}

Authors thank the European Commission (SMETHODS: FP7-ICT-2009-7 Grant Agreement No. 288526, NGCPV: FP7-ENERGY.2011.1.1 Grant Agreement No. 283798), the Spanish Ministries (ENGINEERING METAMATERIALS:

CSD2008-

00066, SEM: TSI-020302-2010-65 SUPERRESOLUCION: TEC2011-24019, SIGMAMODULOS: IPT-2011-1441920000, PMEL: IPT-2011-1212-920000), and UPM (Q090935C59) for the support given to the research activity of the UPM-Optics Engineering Group, making the present work possible.

This work was partly supported by Fundação para a Ciência e Tecnologia, Scholarship SFRH / BD / 80892 / 2011.

\section{REFERENCES}

[1] R. Winston, J. C. Miñano and P. Benítez, Nonimaging Optics, New York, Elsevier-Academic Press, 2005

[2] I. García et al, "Study of non-uniform light profiles on high concentration III-V solar cells using quasi-3D distributed models", Proceeding $33^{\text {rd }}$ IEEE Photovoltaic Specialist Conference, (2008)

[3] M. Victoria et al, "Characterization of the spatial distribution of irradiance and spectrum in concentrating photovoltaic systems and their effect on multi-junction solar cells", Prog. Photovolt: Res. Appl.. doi: 10.1002/pip.1183, (2011)

[4] P. Espinet-González et al, "Triple-Junction Solar Cell Performance Under Fresnel-based Concentrators Taking Into Account Chromatic Aberration And Off-Axis Operation", $8^{\text {th }}$ International Conference on concentrating Photovoltaic systems: CPV-8, AIP Conf. Proc. 1477, pp. 81-84, Toledo, (2012)

[5] R.R. King et al, "Solar cell generations over 40\% efficiency", Prog. Photovolt: Res. Appl.. doi: 10.1002/pip. 1255, (2012)

[6] P. Zamora, et. al. "Experimental characterization of Fresnel-Köhler concentrators," J. Photon. Energy. 2(1), 021806 (2012)

[7] L.W. James, Contractor Report SAND89-7029, (1989)

[8] T. Schult, "Temperature dependence of Fresnel Lens for Concentrated Photovoltaics", Concentrating PV, Darmstadt, (2009) 